\title{
A degree bound for families of rational curves on surfaces
}

\author{
Niels Lubbes \\ July 1, 2021
}

\begin{abstract}
We give an upper bound for the degree of rational curves in a family that covers a given birationally ruled surface in projective space. The upper bound is stated in terms of the degree, sectional genus and arithmetic genus of the surface. We introduce an algorithm for constructing examples where the upper bound is tight. As an application of our methods we improve an inequality on lattice polygons.
\end{abstract}

\section{Contents}

1 Introduction 2

2 Intersection theory 3

3 Adjunction 5

4 Minimal families 10

5 Upper bound for the level 11

6 Algorithm for constructing examples 22

7 Inequality for lattice polygons 28

8 Acknowledgements 30

$\begin{array}{ll}\text { Bibliography } & 30\end{array}$ 


\section{Introduction}

A parametrization of a rational surface $S \subset \mathbb{P}^{n}$ is a birational map

$$
f: \mathbb{C}^{2} \rightarrow S \subset \mathbb{P}^{n}, \quad(s, t) \mapsto\left(f_{0}(s, t): \ldots: f_{n}(s, t)\right) .
$$

The parametric degree of $S$ is defined as the minimum of the set of integers of the form $\max \left\{\operatorname{deg} f_{i} \mid 0 \leq i \leq n\right\}$ for some birational map $f: \mathbb{C}^{2} \rightarrow S$.

An upper bound for the parametric degree over an algebraically closed field of characteristic 0 is given in [10, Theorem 9] in terms of the sectional genus and degree of $S$. In [11, Theorem 20] bounds for the parametric degree over perfect fields are expressed in terms of the level and keel (Definition 1). The upper bound in [10, Theorem 9] can be interpreted as an upper bound on the level. The analysis of [11] applied to toric surfaces led to new inequalities for invariants of lattice polygons in [5]. In [3, Section 2.7] it is conjectured that the inequality can be improved by taking into account the number of vertices. In [6, Theorem 5] these inequalities for lattice polygons are translated to inequalities of rational surfaces and in [6, Section 4] the conjecture of [3] is restated in the context of rational surfaces.

In this paper we generalize the level and keel for rational surfaces in [11, Section 3] to birationally ruled surfaces (this generalization is also posed as an open question in [6, Section 1]). Instead of the parametric degree we now consider the minimal family degree (defined in $\S 4$ ). Theorem 1 gives an upper bound for the level of a birationally ruled surface $S \subset \mathbb{P}^{n}$ in terms of the sectional genus, degree and arithmetic genus. As Corollary 1 we obtain an upper bound for the minimal family degree. If $S$ is rational, then our upper bound for the level coincides with the upper bound for the level in [10, Lemma 8]. However, in order to generalize this bound we give an alternative proof. This proof enables us to make a case distinction on the invariants of $S$, which improves the upper bound for the level. Moreover, these methods enables us to prove the correctness of Algorithm 1 that outputs examples where our upper bound is attained. Thus we show that our upper bound 
for the level is tight in a combinatorial sense. This algorithm is simple but has a non-trivial correctness proof. These methods generalize the inequality [6, Theorem 5] to birationally ruled surfaces. If we restrict our generalized inequality to toric surfaces, we obtain an improved inequality involving lattice polygons as conjectured in [3, Section 2.7],[6, Section 4]. In light of the historical context, one might ask whether this inequality can be improved using the language of lattice geometry.

I would like to end this introduction with some additional remarks on the degree of minimal parametrizations. Let $s(f):=\max \left\{\operatorname{deg}_{s} f_{i} \mid 0 \leq i \leq n\right\}$, $t(f):=\max \left\{\operatorname{deg}_{t} f_{i} \mid 0 \leq i \leq n\right\}$ and we assume without loss of generality that $t(f) \geq s(f)$. The parametric bi-degree of $S$ is defined as the minimum of $(s(f), t(f))$ among all birational maps $f: \mathbb{C}^{2} \rightarrow S$ with respect to the lexicographic order on ordered pairs of integers. If $S \subset \mathbb{P}^{n}$ attains at least two minimal families, then the parametric bi-degree of $S$ equals $(v(S), v(S))$ where $v(S)$ is minimal family degree [7, Theorem 17]. Thus in this case our upper bound for the minimal family degree translates into an upper bound of the parametric bi-degree. If $S$ carries only one minimal family, then an upper bound for the parametric bi-degree is still open. In this case we also have to incorporate the keel in addition to the sectional genus, degree and arithmetic genus of $S$.

\section{Intersection theory}

We recall some intersection theory and this section can be omitted by the expert.

The Neron-Severi group $N(X)$ of a non-singular projective surface $X$ can be defined as the group of divisors modulo numerical equivalence. This group admits a bilinear intersection product

$$
\cdot: N(X) \times N(X) \longrightarrow \mathbb{Z}
$$


The Picard number of $X$ is defined as the rank of $N(X)$. The Neron-Severi theorem states that the Picard number is finite. For proofs in the next section we implicitly also consider $N(X) \otimes \mathbb{R}$. Moreover, we switch between the linear and numerical equivalence class of a divisor where needed.

The class of an exceptional curve $E$ in $N(X)$ is characterized by

$$
E^{2}=E \cdot K=-1,
$$

where $K$ is the canonical divisor class of $X$. Castelnuovo's contractibility criterion states that for all exceptional curves $E$ there exists a contraction map

$$
f: X \longrightarrow Y \text {, }
$$

such that $f(E)=p$ with $p$ a smooth point and $(X \backslash E) \longrightarrow(Y \backslash p)$ is an isomorphism via $f$. The assignment of Neron-Severi groups is functorial such that

$$
f^{*}: N(Y) \longrightarrow N(X)
$$

The groups are related by

$$
N(X) \cong N(Y) \oplus \mathbb{Z}\langle E\rangle,
$$

and thus the Picard number drops for each contracted curve. The formula for pullback of the canonical class is

$$
f^{*}(K)=K_{Y}-E .
$$

Suppose that $D \subset Y$ is a divisor and let $\tilde{D}$ be the strict transform of $D$ along $f: X \longrightarrow Y$. In this case

$$
f^{*}[D]=[\tilde{D}]+m E,
$$

where $[D] \in N(Y),[\tilde{D}] \in N(X)$ and $m$ is the order of $D$ at $p$. For the intersection product we have the projection formula

$$
f^{*}(C) \cdot A=C \cdot f_{*}(A),
$$


and compatibility with the pullback

$$
f^{*}(A) \cdot f^{*}(B)=A \cdot B
$$

for all $A, B \in N(X)$ and $C \in N(Y)$.

The Hodge index theorem states that if $A^{2}>0$ and $A \cdot B=0$, then $B^{2}<0$ or $B=0$ for all $A, B \in N(X)$.

The adjunction formula implies that $A^{2}+A \cdot K \geq-2$ for all $A \in N(X)$. If $D$ is a divisor isomorphic to $\mathbb{P}^{1}$, then $[D]^{2}+[D] \cdot K=-2$ with $[D] \in N(X)$.

We denote by $p_{a}(X)$ the arithmetic genus of $X$ and it is a birational invariant. If $X$ is a ruled surface then $p_{a}(X)$ equals the negative of the geometric genus of its base curve.

The Riemann-Roch theorem states that

$$
h^{0}(D)-h^{1}(D)+h^{2}(D)=\frac{D \cdot(D-K)}{2}+p_{a}(X)+1
$$

for a divisor class $D$ (up to linear equivalence) with associated sheaf $\mathcal{O}(D)$. Here $h^{i}(D)$ denotes the dimension of the i-th sheaf cohomology $\operatorname{dim} H^{i}(X, \mathcal{O}(D))$. Serre duality states that $h^{2}(D)=h^{0}(K-D)$.

\section{Adjunction}

Adjunction works over any field.

We call a divisor class $D$ of a surface efficient if and only if $D \cdot E>0$ for all exceptional curves $E$.

We define a ruled pair as a pair $(X, D)$ where $X$ is a non-singular birationally ruled surface and $D$ is a nef and efficient divisor class of $X$.

If $D$ is effective, then the polarized model of $(X, D)$ is defined as $\overline{\varphi_{D}(X)} \subset$ $\mathbb{P}^{h^{0}(D)-1}$ where $\varphi_{D}$ is the map associated to the global sections $H^{0}(X, \mathcal{O}(D))$. 
If $(X, D)$ is a ruled pair, then $X$ has Kodaira dimension $-\infty$ and thus the canonical divisor class $K$ of $X$ is not nef [9, Section 1.2]. The nef threshold of $D$ is defined as

$$
t(D):=\sup \{q \in \mathbb{R} \mid D+q K \text { is nef }\} .
$$

We call a ruled pair $(X, D)$ non-minimal if $D$ is big and either

(i) $t(D)=1$ and $D \neq-K$, or

(ii) $t(D)>1$.

We call a ruled pair $(X, D)$ minimal if either

(i) $t(D)=1$ and $D=-K$, or

(ii) $t(D)<1$.

An adjoint relation is a relation between two ruled pairs

$$
(X, D) \stackrel{\mu}{\longrightarrow}\left(X^{\prime}, D^{\prime}\right):=\left(\mu(X), \mu_{*}(D+K)\right),
$$

such that $(X, D)$ is a non-minimal ruled pair and $\mu: X \longrightarrow X^{\prime}$ is a birational morphism that contracts all exceptional curves $E$ such that $(D+K) \cdot E=$ 0 .

\section{Lemma 1. (adjoint relation)}

Let $(X, D) \stackrel{\mu}{\longrightarrow}\left(X^{\prime}, D^{\prime}\right)$ be an adjoint relation.

a) $\mu^{*} D^{\prime}=D+K$ and $D^{\prime 2}=(D+K)^{2}$.

b) If $D^{\prime 2}>0$, then $\mu: X \longrightarrow X^{\prime}$ is unique up to biregular isomorphism.

Proof. Let $\left(E_{j}\right)_{j}$ be the curves that are contracted by $\mu: X \longrightarrow X^{\prime}$.

a) See $\S 2$ for the pullback of a divisor class along a contraction map and the compatibility of pullback with the intersection product. From $(D+K) \cdot E_{j}=0$ it follows that $\mu^{*} D^{\prime}=D+K$ and thus $D^{\prime 2}=(D+K)^{2}$. 
b) From the Hodge index theorem, $(D+K)^{2}>0$ and $(D+K) \cdot\left(E_{1}+E_{2}\right)=0$ it follows that $\left(E_{1}+E_{2}\right)^{2}<0$ and thus $E_{1} \cdot E_{2}=0$. It follows that if $D^{\prime 2}>0$, then the contracted exceptional curves are disjoint. The contraction of an exceptional curve is an isomorphism outside this exceptional curve. Thus the order of contracting disjoint curves does not matter up to biregular isomorphism.

\section{Proposition 1. (adjoint relation)}

If $(X, D) \stackrel{\mu}{\longrightarrow}\left(X^{\prime}, D^{\prime}\right)$ is an adjoint relation, then $\left(X^{\prime}, D^{\prime}\right)$ is either a nonminimal or a minimal ruled pair.

Proof. We use the pullback formulas for divisor classes, its compatibility with the intersection product and the projection formula as described in $\S 2$.

Suppose by contradiction that $D^{\prime}$ is not nef. It follows that there exists a curve $C^{\prime}$ such that $D^{\prime} \cdot C^{\prime}=\mu^{*} D^{\prime} \cdot \mu^{*} C^{\prime}<0$. From $\mu^{*} D^{\prime} \cdot \mu^{*} C^{\prime}<0$ and Lemma 1.a) it follows that $(D+K) \cdot C<0$, where $C$ is the strict transform of $C^{\prime}$. However, the nef threshold $t(D)$ is greater or equal to one. We have thus arrived at a contradiction.

Suppose by contradiction that $D^{\prime}$ is not efficient. From Lemma 1.a) it follows that there exists exceptional curve $E^{\prime}$ such that $D^{\prime} \cdot E^{\prime}=\mu^{*} D^{\prime} \cdot \mu^{*} E^{\prime}=$ $(D+K) \cdot E=0$ where $E$ is the strict transform of $E^{\prime}$. We find that $K^{\prime} \cdot \mu_{*} E=$ $\mu^{*} K^{\prime} \cdot E=-1$ and thus $K \cdot E \leq-1$. From

$\mu^{*} K^{\prime} \cdot \mu^{*} E^{\prime}=\left(K-\sum_{j} E_{j}\right) \cdot\left(E+\sum_{j} m_{j} E_{j}\right)=K \cdot E-\sum_{a \neq b} m_{a} E_{a} \cdot E_{b}=-1$,

it follows that $K \cdot E \geq-1$. From the adjunction formula and $E \cong \mathbb{P}^{1}$ it follows that $E^{2}+E \cdot K=-2$. Therefore $E^{2}=E \cdot K=-1$ and thus $E$ is an exceptional curve not contracted by $\mu$. We arrived at a contradiction.

We call a minimal ruled pair $(X, D)$ a weak Del Pezzo pair if and only if either $D=-K, D=-\frac{1}{2} K, D=-\frac{1}{3} K$, or $D=-\frac{2}{3} K$, with $K$ the canonical divisor class of $X$. 
We call a minimal ruled pair $(X, D)$ a geometrically ruled pair if and only if $\varphi_{M}: X \longrightarrow C$ is a geometrically ruled surface such that either $M=a D$, or $M=a(2 D+K)$ for large enough $a \in \mathbb{Z}_{>0}$. Here $\varphi_{M}$ is the map associated to the global sections $H^{0}(X, \mathcal{O}(M)), C=\varphi_{M}(X)$ and $K$ is the canonical divisor class of $X$.

\section{Proposition 2. (Neron-Severi group of a minimal ruled pair)}

Let $(X, D)$ be a minimal ruled pair, with $K$ be the canonical divisor class of $X$ and $N(X)$ the Neron-Severi group. Let $p$ denote the arithmetic genus of $X$.

a) If $(X, D)$ is a weak Del Pezzo pair with $K^{2} \neq 8$, then $p=0$, $N(X) \cong \mathbb{Z}\left\langle H, Q_{1}, \ldots, Q_{r}\right\rangle$ with $0 \leq r=9-K^{2} \leq 8$ and intersection product $H \cdot Q_{i}=0, Q_{i}^{2}=1$ and $Q_{i} \cdot Q_{j}=0$ for $i \neq j$ in $[1, r]$. We have $-K=3 H-Q_{1}-\ldots-Q_{r}$ and either $D=-K, D=-\frac{1}{3} K$, or $D=-\frac{2}{3} K$.

b) If $(X, D)$ is a weak Del Pezzo pair with $K^{2}=8$, then $p=0, N(X) \cong$ $\mathbb{Z}\langle H, F\rangle$ with intersection product $H^{2}=r, H \cdot F=1$ and $F^{2}=0$ for $r \in\{0,1,2\}$. We have $K=-2 H+(r-2) F$ and either $D=-K$ or $D=-\frac{1}{2} K$.

c) If $(X, D)$ is a geometrically ruled pair, then $N(X) \cong \mathbb{Z}\langle H, F\rangle$ with intersection product $H^{2}=r, H \cdot F=1$ and $F^{2}=0$ for $r \in \mathbb{Z}_{\geq 0}$. Either $D=k F$ or $2 D+K=k F$ for $k \in \mathbb{Z}_{>0}$ and $K=-2 H+(r-2 p-2) F$ such that $K^{2}=8(p+1)$.

Proof. For a) and b) see [4, Section 8.4.3]. For c) see [2, Chapter 3, Proposition 18, page 34].

An adjoint chain of a ruled pair is defined as a chain of successive adjoint relations until a minimal ruled pair is obtained. 


\section{Proposition 3. (adjoint chain)}

An adjoint chain must terminate and a minimal ruled pair at the end is either a weak Del Pezzo pair or a geometrically ruled pair.

Proof. Let $(X, D)$ be a non-minimal ruled pair and let $t:=t(D)$ be the nef threshold. Let $K$ be the canonical class of $X$. From [9, Corollary 1-2-15] it follows that $t \in \mathbb{Q}_{>0}$ with denominator bounded by 3. After a $\lfloor t\rfloor$ successive adjoint relations $D$ is replaced by the pushforward of $D+\lfloor t\rfloor K$ and thus we may assume that $t \leq 1$. We make a case distinction.

First suppose that $(D+t K)^{2}>0$. There exists an irreducible curve $C$ such that $(D+t K) \cdot C=0, D \cdot C>0$ and $K \cdot C<0$. From the Hodge index theorem and $(D+t K)^{2}>0$ it follows that $C^{2}<0$. From the adjunction formula it follows that $C^{2}+K \cdot C=-2$. From [9, Lemma 1-1-4] it follows that $C$ is an exceptional curve. Recall from $\S 2$ that the Picard number drops for each contracted exceptional curve and that this number is finite.

Next, if $(D+t K)^{2}=0$ and $D=-t K$, then $(X, D)$ is a weak Del Pezzo pair.

Finally, we assume that $(D+t K)^{2}=0$ and $D \neq-t K$. If $t=1$, then we apply one extra adjoint relation so we may assume that $t<1$. From [9, Theorem 1-2-14 and Proposition 1-2-16] it follows that that the map associated to $a(D+t K)$, with large enough $a \in \mathbb{Z}_{>0}$, defines a Mori fibre space [9, Definition 1-4-1]. It follows from [9, Theorem 1-4-4] that a fibre $F$ of this morphism is isomorphic to $\mathbb{P}^{1}$ with $F^{2}=0$. By the adjunction formula we have $F \cdot K=-2$. From $(D+t K) \cdot F=0$ it follows that $t=\frac{D \cdot F}{2} \in \frac{1}{2} \mathbb{Z}_{\geq 0}$. Thus in this case $(X, D)$ is a geometrically ruled pair.

\section{Definition 1. (level and keel)}

Suppose $\left(X_{0}, D_{0}\right) \stackrel{\mu_{0}}{\longrightarrow}\left(X_{1}, D_{1}\right) \stackrel{\mu_{1}}{\longrightarrow} \ldots \stackrel{\mu_{\ell-1}}{\longrightarrow}\left(X_{\ell}, D_{\ell}\right)$ is an adjoint chain.

The level of $\left(X_{0}, D_{0}\right)$ is defined by $\ell$. The keel of $\left(X_{0}, D_{0}\right)$ is either:

- 0 if $\left(X_{\ell}, D_{\ell}\right)$ is a weak Del Pezzo pair, or

- $k$ as in Proposition 2.c) if $\left(X_{\ell}, D_{\ell}\right)$ is a geometrically ruled pair. $\triangleleft$ 


\section{Proposition 4. (level and keel)}

The level and keel are well defined.

Proof. Let $(X, D) \stackrel{\mu}{\longrightarrow}\left(X^{\prime}, D^{\prime}\right)$ be an adjoint relation.

Since $D^{\prime 2}=0$ can only occur at the last adjoint relation in an adjoint chain, it follows from Lemma 1.b) that the level is well defined.

We now show that also the keel does not depend on the last adjoint relation and thus is uniquely defined. Suppose that $\left(X^{\prime}, D^{\prime}\right)$ is a geometrically ruled pair. From Proposition 2 and Lemma 1.a) it follows that if $D^{\prime 2}=(D+K)^{2}=$ 0 , then $-2 k=\mu^{*} D^{\prime} \cdot \mu^{*} K^{\prime}=(D+K) \cdot K$ defines the keel $k$. Similarly, if $D^{\prime 2}=(D+K)^{2}>0$, then $-2 k=\mu^{*}\left(2 D^{\prime}+K^{\prime}\right) \cdot \mu^{*} K^{\prime}=2(D+K) \cdot K+K^{\prime 2}$. From Proposition 2 it follows that $K^{\prime 2}=8(p+1)$, where the arithmetic genus $p$ is a birational invariant. Thus our assertion holds.

\section{Remark 1. (level and keel)}

The level and keel have been introduced in [11, Section 3]. In our generalization to birationally ruled surfaces we use a slightly alternative definition for the level, since it simplifies our arguments. If $D_{\ell}=-K_{\ell}, D_{\ell}=-\frac{1}{2} K_{\ell}$, $D_{\ell}=-\frac{1}{3} K_{\ell}, D_{\ell}=-\frac{2}{3} K_{\ell}, D_{\ell}=k F$ or $2 D_{\ell}+K_{\ell}=k F$ as in Proposition 2, then we define $\lambda$ as $1, \frac{1}{2}, \frac{1}{3}, \frac{2}{3}$, 0 or $\frac{1}{2}$ respectively. Now the level in [11, Section 3] for rational surfaces is defined as $\ell+\lambda$.

\section{Minimal families}

A family of curves $F$ for ruled pair $(X, D)$ that is indexed by a smooth curve $C$, is defined as a divisor $F \subset X \times C$ such that the first projection $F \longrightarrow X$ is dominant. If the generic curve of $F$ is rational and if $D \cdot F$ is minimal with respect to all families of rational curves, then we call $F$ minimal. The minimal family degree $v(X, D)$ is defined as $D \cdot F$ for a minimal family $F$. Note that since $(X, D)$ is a ruled pair, there always exists a minimal 
family.

We recall part of [8, Theorem 46] concerning the degree of minimal families along an adjoint relation $(X, D) \stackrel{\mu}{\longrightarrow}\left(X^{\prime}, D^{\prime}\right)$. If $X \cong X^{\prime} \cong \mathbb{P}^{2}$, then $v(X, D)=v\left(X^{\prime}, D^{\prime}\right)+3$, else $v(X, D)=v\left(X^{\prime}, D^{\prime}\right)+2$. If $(X, D)$ is a weak Del Pezzo pair and $X \cong \mathbb{P}^{2}$, then $v(X, D) \leq 3$. If $(X, D)$ is a weak Del Pezzo pair and $D^{2}=8$, then $v(X, D) \leq 2$. If $(X, D)$ is a weak Del Pezzo pair and $D^{2}<8$, then $v(X, D)=2$. If $(X, D)$ is a geometrically ruled pair, then $v(X, D) \leq 1$.

\section{Upper bound for the level}

\section{1}

Let $\left(X_{0}, D_{0}\right) \stackrel{\mu_{0}}{\longrightarrow}\left(X_{1}, D_{1}\right) \stackrel{\mu_{1}}{\longrightarrow} \ldots \stackrel{\mu_{\ell-1}}{\longrightarrow}\left(X_{\ell}, D_{\ell}\right)$ be an adjoint chain. From now on let $K_{i}$ denote the canonical class of $X_{i}$. We introduce the following notation:

$$
\alpha(i)=D_{i}^{2}, \quad \beta(i)=D_{i} \cdot K_{i}, \quad \gamma(i)=K_{i}^{2}, \quad h(i)=D_{i}^{2}-D_{i} \cdot K_{i}
$$

and $n(i)$ denotes the number of curves contracted by $\mu_{i}$ for $0 \leq i \leq \ell$.

\section{Lemma 2. (adjoint intersection products)}

If $\ell>0$, then

a) $\alpha(i+1)=\alpha(i)+2 \beta(i)+\gamma(i)$,

b) $\beta(i+1)=\beta(i)+\gamma(i)$,

c) $\gamma(i+1)=\gamma(i)+n(i)$,

d) $h(i+1)=h(i)+2 \beta(i)$,

for $0 \leq i \leq \ell-1$. 
Proof. We use the pullback formulas for divisor classes, its compatibility with the intersection product and the projection formula as described in $\S 2$. Now a) and b) are a straightforward consequence of Lemma 1.a). Let $\left(E_{j}\right)_{j}$ be the curves that are contracted by $\mu_{i}: X_{i} \longrightarrow X_{i+1}$. For c) we compute

$$
K_{i}^{2}=\left(\mu_{i}^{*} K_{i+1}\right)^{2}=K_{i+1}^{2}-n(i)+\sum_{j \neq k} E_{j} \cdot E_{k},
$$

and we need to show that $\sum_{j \neq k} E_{j} \cdot E_{k}=0$. From $\mu_{i *} E_{k}=0$ it follows that $K_{i+1} \cdot \mu_{i *} E_{k}=0$ and thus

$K_{i+1} \cdot \mu_{i *} E_{k}=\mu_{i}^{*} K_{i+1} \cdot E_{k}=\left(K_{i}-\sum_{j} E_{j}\right) \cdot E_{k}=-1+1-\sum_{j \neq k} E_{j} \cdot E_{k}=0$.

This proves c). From $h(i+1)=\alpha(i+1)-\beta(i+1)=\alpha(i)+\beta(i)=h(i)+2 \beta(i)$ it follows that $\mathrm{d}$ ) holds.

Remark 2. Lemma 2.d) is essentially [10, Lemma 7], which is attributed there to Castelnuovo [10, Remark 3].

We say that $\left(X_{i}, D_{i}\right)$ has adjoint state $S_{a}(i)$ for some $1 \leq a \leq 4$ if $\gamma(i)$ and $\beta(i)$ are as in the following table:

\begin{tabular}{|c|c|c|}
\hline adjoint state & $\gamma(i)$ & $\beta(i)$ \\
\hline \hline$S_{1}(i)$ & $<0$ & $\geq 0$ \\
\hline$S_{2}(i)$ & $<0$ & $<0$ \\
\hline$S_{3}(i)$ & $=0$ & $<0$ \\
\hline$S_{4}(i)$ & $>0$ & $<0$ \\
\hline
\end{tabular}

where $0 \leq i \leq \ell$.

\section{Lemma 3. (adjoint states)}

a) A ruled pair $\left(X_{i}, D_{i}\right)$ has adjoint state either $S_{1}(i), S_{2}(i), S_{3}(i)$ or $S_{4}(i)$ for all $0 \leq i \leq \ell$. 
b) If $S_{a}(i)$ and $S_{b}(i+1)$ for $0 \leq i \leq \ell-1$, then $a \leq b$.

Proof.

a) We assume first that $\gamma(i)=0$. Assume by contradiction that $\beta(i) \geq 0$. From Lemma 2 it follows that $\alpha(j+1) \geq \alpha(j)$ and $\beta(j)=\beta(j+1)$ for all

$j \geq i$. But then the adjoint chain is of infinite length. We have thus arrived at a contradiction.

Next we assume that $\gamma(i)>0$. From Proposition 2 it follows that $p=$ $\min \left(0,\left\lceil\frac{1}{8} \gamma(\ell)-1\right\rceil\right)$ and thus $p=0$. From the Riemann Roch theorem and Serre duality it follows that $h^{0}\left(-K_{i}\right) \geq \gamma(i)+1>0$. From $D_{i}$ being nef it follows that $\beta(i) \leq 0$. From the Hodge index theorem it follows that $\beta(i)<0$.

b) From Lemma 2 it follows that $\gamma(i)<\gamma(i+1)$ and if $\gamma(i)<0$, then $\beta(i+1)<\beta(i)$.

Lemma 4. (dimension)

We have that $h(i) \geq 2$ for $1 \leq i \leq \ell$.

Proof. By Lemma 2 we have $h(0)+h(1)=2 \alpha(0)>0$. Thus $h(0)>0$ and the base case $h(1)>0$ of the induction holds. By induction hypothesis $h(i)>0$. The induction step is to show that $h(i+1)>0$. If $\beta(i) \geq 0$, then from Lemma 2 it follows that $h(i+1)=h(i)+2 \beta(i)>0$. If $\beta(i)<0$, then by Lemma 3 we have $\beta(i+1)<0$ and thus $h(i+1)=\alpha(i+1)-\beta(i+1)>0$. We can conclude from the Riemann-Roch theorem that $h(i)$ must be even and thus $h(i) \neq 1$.

\section{2}

We will now consider the following combinatorial problem. For given

$$
h(0), \beta(0), p \in \mathbb{Z},
$$


find an upper bound for $\ell$ such that there exists a sequence of integer 3tuples

$$
(h(i), \beta(i), \gamma(i))_{0 \leq i \leq \ell},
$$

which adheres to the following 5 rules:

(H) $h(i+1)=h(i)+2 \beta(i)($ Lemma 2.d $)$.

(B) $\beta(i+1)=\beta(i)+\gamma(i)($ Lemma 2.b)).

(Z) $h(i) \geq 2$ for $1 \leq i \leq l$ (Lemma 4$)$.

(S) $S_{a}(i)$ for $1 \leq a \leq 4$ and if $S_{a}(i)$ and $S_{b}(i+1)$, then $a \leq b$ (Lemma 3 ).

(P) $p \leq 0$. If $p=0$, then $\gamma(\ell)>0$. If $p<0$, then $\gamma(\ell)=8(p+1)$. (Proposition 2).

Using Proposition 2 it is possible to impose restrictions on $(\gamma(\ell), \beta(\ell))$ and from the Riemann-Roch theorem we can conclude that $h(i)$ must be even. However, we do not need these additional rules for a proof. For our solution of the posed problem we make a case distinction between $S_{1}(0), S_{2}(0), S_{3}(0)$ and $S_{4}(0)$. In the proof of Theorem 1 we will compose the upper bounds of these cases.

First we start with a technical lemma for convenience.

Lemma 5. (technical lemma)

$$
\begin{aligned}
& \text { If } \gamma(0)=\ldots=\gamma(j-1) \text { for some } 1 \leq j \leq \ell \text {, then } \\
& \qquad h(j)=\gamma(0) j^{2}+(2 \beta(0)-\gamma(0)) j+h(0) .
\end{aligned}
$$

Proof. With (H) we expand $h(j)$ such that

$$
h(j)=h(j-1)+2 \beta(j-1)=\ldots=h(0)+2 \sum_{n=0}^{j-1} \beta(n) .
$$

With (B) we expand the $\beta(i)$ terms such that

$$
h(j)=h(0)+2 \sum_{n=0}^{j-1}(\beta(0)+n \gamma(0))=h(0)+2 j \beta(0)+j(j-1) \gamma(0) .
$$


We conclude this proof by re-arranging terms.

Lemma 6. (case $S_{4}(0)$ )

If $S_{4}(0)$, then

$$
\ell \leq-\beta(0)-1<\frac{h(0)-2}{2}
$$

Proof. From (S) and (B) it follows that $\beta(0)<\ldots<\beta(\ell)<0$ and thus we conclude the first inequality. The second inequality follows from $h(0)+$ $2 \beta(0) \geq 2$.

\section{Lemma 7. (case $S_{3}(0)$ )}

If $S_{3}(0)$, then

$$
\ell \leq \frac{h(0)-2}{2}
$$

Moreover, if $\ell$ is equal to this upper bound, then $S_{3}(\ell-1)$.

Proof. It follows from $(\mathrm{H})$ and $(\mathrm{S})$ that $h(i+1)-h(i)=2 \beta(i) \leq-2$ if $S_{3}(i)$ or $S_{4}(i)$ for all $0 \leq i \leq \ell$. The upper bound asserted in the lemma now follows from $(\mathrm{Z})$. This upper bound is attained if $\beta(i)=-1$ for $0 \leq i \leq \ell$. It follows that $S_{4}(i)$ if and only if $i=\ell$ and $p=0$. Thus we can conclude from $(\mathrm{S})$ that $S_{3}(\ell-1)$ in case of equality.

For an example where the upper bound of Lemma 7 is attained, see $3 \leq i \leq 7$ in Table 1 of Example 1.

Lemma 8. (case $S_{2}(0)$ with $p \geq-1$ )

If $S_{2}(0)$ and $p \geq-1$, then

$$
\ell<\frac{h(0)-2}{2}
$$

If $\beta(0)-\beta(\ell)>0$, then

$$
\ell \leq s+\left\lfloor\frac{-s^{2}+(2 \beta(0)+1) s+h(0)-2}{-2 \beta(\ell)}\right\rfloor,
$$

where $s:=\beta(0)-\beta(\ell)$. 
Proof. Suppose that $s>0$. It follows from (S) and (B) that if $S_{3}(k)$ or $S_{4}(k)$, then $\beta(k) \leq \beta(\ell)$ for $0 \leq k \leq \ell$. From (B) it follows that $k \geq s$ where we have equality if $\gamma(0)=\ldots=\gamma(k-1)=-1$. It now follows from Lemma 5 that

$$
h(k) \leq h(s)=-s^{2}+(2 \beta(0)+1) s+h(0) .
$$

It follows from (S) that $\beta(i)<0$ for $k \leq i \leq \ell$. From (Z) and thus the same argument in Lemma 7 it follows that

$$
\ell \leq s+\frac{h(s)-2}{-2 \beta(\ell)} .
$$

The first inequality follows if $\beta(0)=\beta(\ell)=-1$ such that $s=0$.

For an example where the second upper bound of Lemma 8 is attained, see $6 \leq i \leq 12$ in Table 2 of Example 1.

Lemma 9. (case $S_{2}(0)$ with $p \leq-2$ )

If $S_{2}(0)$ with $p \leq-2$, then

$$
\ell \leq\left\lfloor\frac{-(2 \beta(0)-t)-\sqrt{\Delta}}{2 t}\right\rfloor,
$$

where $\Delta=(2 \beta(0)-t)^{2}-4 t(h(0)-2)$ and $t:=8(p+1)$.

Proof. From (P) and (S) it follows that $\gamma(\ell)=8(p+1)$ and $S_{2}(\ell)$. From $(\mathrm{Z})$ and $(\mathrm{H})$ it follows that $h(\ell)+2 \beta(\ell) \leq 0$. It follows from $(\mathrm{H})$ that $h(i)$ decreases as slow as possible if $\gamma(0)=\ldots=\gamma(\ell)$. It follows from (Z) that $h(\ell) \geq 2$ so that we can equate the formula of Lemma 5 to 2 . The upper bound for $\ell$ now follows from the quadratic formula.

For an example where the upper bound of Lemma 9 is attained, see $5 \leq i \leq 9$ in Table 4 of Example 1. 
Lemma 10. (case $S_{1}(0)$ )

If $S_{1}(0)$ and $j$ is the largest index such that $S_{1}(j-1)$, then

$$
j \leq\left\lfloor\frac{\beta(0)}{-t}\right\rfloor+1
$$

and

$$
h(j) \leq t\left(\left\lfloor\frac{\beta(0)}{-t}\right\rfloor+1\right)^{2}+(2 \beta(0)-t)\left(\left\lfloor\frac{\beta(0)}{-t}\right\rfloor+1\right)+h(0),
$$

where

$$
t:=\min (8(p+1),-1) .
$$

Moreover, if the upper bound for $j$ and $h(j)$ is reached, then $\beta(j)=t$.

In case $p \geq-1$ then the upper bound for $h(j)$ simplifies to

$$
h(j) \leq \beta(0)^{2}+\alpha(0) .
$$

Proof. From $(\mathrm{S})$ and $(\mathrm{P})$ we find that $\gamma(i) \leq t$ for $i<j$. It follows from $(\mathrm{S})$ and (B) that in order to find an upper bound for $j$ we need to assume that $\gamma(0)=\ldots=\gamma(j-1)=t$ such that

$$
\beta(j-1)=\beta(j-2)-\gamma(0)=\ldots=\beta(0)-(j-1) \gamma(0)=0 .
$$

From this we conclude the upper bound for $j$. The upper bound for $h(j)$ is reached if we substitute the upper bound for $j$ in the formula of Lemma 5 . From (B) it follows that $\beta(j)=t$ if the upper bounds for $j$ and $h(j)$ are

reached. If $p \geq-1$, then $t$ divides $\beta(0)$ so that this formula simplifies.

For an example where upper bound of Lemma 10 is attained, see $0 \leq i \leq 2$ in Table 1 of Example 1.

\section{Theorem 1. (upper bound level)}

We state upper bounds for the level in terms of $\alpha(0), \beta(0)$ and $p$, where $p$ is the arithmetic genus of $X_{0}$. 
If $p=0$ or $p=-1$, then

$$
\ell \leq \frac{\beta(0)^{2}+\alpha(0)}{2}+\beta(0),
$$

and if moreover $\beta(0)<0$, then

$$
\ell \leq \frac{\alpha(0)-\beta(0)-2}{2} .
$$

If $p \leq-2$, then

$$
\ell \leq\left\lfloor\frac{\beta(0)}{-t}\right\rfloor+1+\left\lfloor\frac{-t-\sqrt{t^{2}-4 t(\Upsilon-2)}}{2 t}\right\rfloor,
$$

and if moreover $\beta(0)<0$, then

$$
\ell \leq\left\lfloor\frac{-(2 \beta(0)-t)-\sqrt{\Delta}}{2 t}\right\rfloor
$$

where

$$
t:=8(p+1) \quad \text { and } \quad \Delta:=(2 \beta(0)-t)^{2}-4 t(\alpha(0)-\beta(0)-2),
$$

and

$$
\Upsilon:=t\left(\left\lfloor\frac{\beta(0)}{-t}\right\rfloor+1\right)^{2}+(2 \beta(0)-t)\left(\left\lfloor\frac{\beta(0)}{-t}\right\rfloor+1\right)+\alpha(0)-\beta(0) .
$$

Proof. Recall that by definition $h(0)=\alpha(0)-\beta(0)$. Thus in order to proof this theorem we need to solve the problem as posed at the beginning of $§ 5.2$.

First we assume that $-1 \leq p \leq 0$. It follows from $(\mathrm{S})$ that an upper bound of $\ell$ is obtained as the composition of the upper bound of Lemma 10 with the upper bound of either Lemma 8, Lemma 7 or Lemma 6. It follows that the upper bound of Lemma 7 is the choice which acquires the highest upper bound. If $\beta(0)<0$, then we can apply the upper bound of Lemma 7 directly. If $p \leq-2$, then it follows from $(\mathrm{S})$ and $(\mathrm{P})$ that the upper bound as asserted in this theorem can be obtained by composing the upper bound of Lemma 10 with the upper bound in Lemma 9 . If $\beta(0)<0$, then we can apply the upper bound of Lemma 9 directly. 


\section{Corollary 1. (upper bound for the minimal family degree)}

Let $v=v\left(X_{0}, D_{0}\right)$ be the minimal family degree. Let $\tilde{\ell}$ be the upper bound for the level from Theorem 1.

If $p=0$, then $v \leq 2 \tilde{\ell}+2$.

If $p \leq-1$, then $v \leq 2 \tilde{\ell}+1$.

Proof. We recall from $\S 4$ that if $X_{i} \cong X_{i+1} \cong \mathbb{P}^{2}$, then $p=0$ and

$$
v(i+1)=v(i)+3,
$$

where $v(i):=v\left(X_{i}, D_{i}\right)$. Otherwise $v(i+1)=v(i)+2$. We want to show that $3 \ell+3<2 \tilde{\ell}+2$ and thus we may assume that the minimal family degree is increased by 2 at each step.

First we observe that if $X_{i} \cong \mathbb{P}^{2}$, then $\gamma(i)=9$ and thus $S_{4}(i)$ for all $0 \leq i \leq \ell$. So we may assume without loss of generality that $S_{4}(0)$. Suppose that $X_{i} \cong \mathbb{P}^{2}$ for $0 \leq i \leq \ell$ such that $v(i+1)=v(i)+3$ at each step. In this case it follows from Proposition 2 that $v(\ell) \leq 3$ and $\beta(\ell) \leq-3$. It follows from $(\mathrm{H})$ and $(\mathrm{Z})$ that

$$
\ell \leq \frac{h(0)-2}{6} \text { and thus } v \leq 3\left(\frac{h(0)-2}{6}\right)+3 .
$$

For the upper bound $\tilde{\ell}$ we may assume without loss of generality that $\beta(0)<0$ since if $S_{1}(i)$, then $X_{i} ¥ \mathbb{P}^{2}$ for all $0 \leq i \leq \ell$. From Theorem 1 we used Lemma 7 and thus assumed $S_{3}(i)$ with $\beta(i)=\beta(\ell)=-1$ for $0 \leq i \leq \ell-1$. In this case it follows from Proposition 2 that $v(\ell) \leq 2$. It follows that

$$
\ell \leq \tilde{\ell}=\frac{h(0)-2}{2} \text { and thus } \quad v \leq 2\left(\frac{h(0)-2}{2}\right)+2
$$

Thus indeed we established that $3 \ell+3<2 \tilde{\ell}+2$. We conclude this proof by recalling from $\S 4$ that if $p \leq-1$, then $v(\ell) \leq 1$. 


\section{Remark 3. (computing invariants)}

Note that $\alpha(0)$ is the degree of the (projection of the) polarized model of $\left(X_{0}, D_{0}\right)$. From the adjunction formula it follows that the geometric genus of a generic hyperplane section of $\left(X_{0}, D_{0}\right)$ is equal to the arithmetic genus

$$
p_{a}\left(D_{0}\right)=\frac{\alpha(0)+\beta(0)}{2}+1
$$

It follows that $\alpha(0)$ and $\beta(0)$ can be computed from the degree and geometric genus of a generic hyperplane section. Let $Y \subset \mathbb{P}^{n}$ be the polarized model of $\left(X_{0}, D_{0}\right)$, so that $Y$ is linearly normal. From Proposition 3 it follows that

$$
n+1=h^{0}\left(D_{0}\right)=\frac{\alpha(0)-\beta(0)}{2}+p+1
$$

and thus we can compute the arithmetic genus $p$ of $X_{0}$.

\section{Example 1. (adjoint chains)}

In the following four tables we represent the invariants from $§ 5.1$ that follow the combinatorics of adjoint chains. We denote the upper bound of Theorem 1 by $\tilde{\ell}(i)$. See the beginning of $\S 5.1$ for the remaining notation. The heading of each table denotes the arithmetic genus of $X_{0}$ and the number of different adjoint states that are reached. The transition between adjoint states is indicated by a vertical double line. These examples confirm that the upper bounds in Theorem 1 and Corollary 1 are tight with respect to the combinatorics. The tables were constructed using Algorithm 1 (see forward).

In Table 1 the minimal pair is a weak Del Pezzo pair of degree 1. The upper bound for the level is tight for this example and it follows the analysis of the proof of Theorem 1. The polarized model of this surface is of degree 8. From $\S 4$ it follows that $v\left(X_{0}\right)=18$.

In Table 2 the minimal pair is a weak Del Pezzo pair of degree 3. We see that the upper bound for the level is not tight in this example. All the adjoint states are reached in this example. If the arithmetic genus is zero, then the

upper bound is tight if adjoint state $S_{2}$ is not attained, as was the case in Table 1. 
In Table 3 the minimal pair is a geometrically ruled surface such that $p=-1$ and $2 D+K=k F$ as in Proposition 2. We find that the upper bound for the level in Theorem 1 is tight. The upper bound for the minimal family degree in Corollary 1 is also tight: $v\left(X_{0}\right)=17$.

In Table 4 the minimal pair is a geometrically ruled surface such that $p=-2$ and $D=k F$ as in Proposition 2. We find that the upper bound for the level is tight. From Corollary 1 it follows that $v\left(X_{0}\right) \leq 19$. From $\S 4$ and $\alpha(\ell)=0$ it follows that $v\left(X_{0}\right)=18$.

Table 1 (arithmetic genus 0 and 3 adjoint states)

\begin{tabular}{|l||r|r|r||r|r|r|r|r||r|}
\hline$i$ & 0 & 1 & 2 & 3 & 4 & 5 & 6 & 7 & 8 \\
\hline \hline$n(i)$ & 0 & 0 & 1 & 0 & 0 & 0 & 0 & 1 & \\
\hline$\gamma(i)$ & -1 & -1 & -1 & 0 & 0 & 0 & 0 & 0 & 1 \\
\hline$\beta(i)$ & 2 & 1 & 0 & -1 & -1 & -1 & -1 & -1 & -1 \\
\hline$h(i)$ & 6 & 10 & 12 & 12 & 10 & 8 & 6 & 4 & 2 \\
\hline$\alpha(i)$ & 8 & 11 & 12 & 11 & 9 & 7 & 5 & 3 & 1 \\
\hline$\tilde{\ell}(i)$ & 8 & 7 & 6 & 5 & 4 & 3 & 2 & 1 & 0 \\
\hline
\end{tabular}

Table 2 (arithmetic genus 0 and 4 adjoint states)

\begin{tabular}{|l||r|r|r|r|r|r||r|r||r|r|r|r||r|}
\hline$i$ & 0 & 1 & 2 & 3 & 4 & 5 & 6 & 7 & 8 & 9 & 10 & 11 & 12 \\
\hline \hline$n(i)$ & 0 & 0 & 0 & 0 & 0 & 0 & 0 & 1 & 0 & 0 & 0 & 3 & \\
\hline$\gamma(i)$ & -1 & -1 & -1 & -1 & -1 & -1 & -1 & -1 & 0 & 0 & 0 & 0 & 3 \\
\hline$\beta(i)$ & 5 & 4 & 3 & 2 & 1 & 0 & -1 & -2 & -3 & -3 & -3 & -3 & -3 \\
\hline$h(i)$ & 6 & 16 & 24 & 30 & 34 & 36 & 36 & 34 & 30 & 24 & 18 & 12 & 6 \\
\hline$\alpha(i)$ & 11 & 20 & 27 & 32 & 35 & 36 & 35 & 32 & 27 & 21 & 15 & 9 & 3 \\
\hline$\tilde{\ell}(i)$ & 23 & 22 & 21 & 20 & 19 & 18 & 17 & 8 & 4 & 3 & 2 & 1 & 0 \\
\hline
\end{tabular}


Table 3 (arithmetic genus -1 and 2 adjoint states)

\begin{tabular}{|l||r|r|r||r|r|r|r|r|r|}
\hline$i$ & 0 & 1 & 2 & 3 & 4 & 5 & 6 & 7 & 8 \\
\hline \hline$n(i)$ & 0 & 0 & 1 & 0 & 0 & 0 & 0 & 0 & \\
\hline$\gamma(i)$ & -1 & -1 & -1 & 0 & 0 & 0 & 0 & 0 & 0 \\
\hline$\beta(i)$ & 2 & 1 & 0 & -1 & -1 & -1 & -1 & -1 & -1 \\
\hline$h(i)$ & 6 & 10 & 12 & 12 & 10 & 8 & 6 & 4 & 2 \\
\hline$\alpha(i)$ & 8 & 11 & 12 & 11 & 9 & 7 & 5 & 3 & 1 \\
\hline$\tilde{\ell}(i)$ & 8 & 7 & 6 & 5 & 4 & 3 & 2 & 1 & 0 \\
\hline
\end{tabular}

Table 4 (arithmetic genus -2 and 2 adjoint states)

\begin{tabular}{|l||r|r|r|r|r||r|r|r|r|r|}
\hline$i$ & 0 & 1 & 2 & 3 & 4 & 5 & 6 & 7 & 8 & 9 \\
\hline \hline$n(i)$ & 0 & 0 & 0 & 0 & 0 & 0 & 0 & 0 & 0 & \\
\hline$\gamma(i)$ & -8 & -8 & -8 & -8 & -8 & -8 & -8 & -8 & -8 & -8 \\
\hline$\beta(i)$ & 32 & 24 & 16 & 8 & 0 & -8 & -16 & -24 & -32 & -40 \\
\hline$h(i)$ & 40 & 104 & 152 & 184 & 200 & 200 & 184 & 152 & 104 & 40 \\
\hline$\alpha(i)$ & 72 & 128 & 168 & 192 & 200 & 192 & 168 & 128 & 72 & 0 \\
\hline$\tilde{\ell}(i)$ & 9 & 8 & 7 & 6 & 5 & 4 & 3 & 2 & 1 & 0 \\
\hline
\end{tabular}

\section{Algorithm for constructing examples}

\section{1}

We continue to use the same notation as in the previous section. The following lemma expresses the invariants of the first ruled pair in an adjoint chain in terms of $n(i)$ and the invariants of the minimal pair. 
Lemma 11. (formulas for intersection products)

$$
\begin{aligned}
& \text { a) } \alpha(0)=\gamma(\ell) \ell^{2}-2 \beta(\ell) \ell+\alpha(\ell)-\sum_{i=0}^{\ell-1}(i+1)^{2} n(i) \text {. } \\
& \text { b) } \beta(0)=-\gamma(\ell) \ell+\beta(\ell)+\sum_{i=0}^{\ell-1}(i+1) n(i) . \\
& \text { c) } \gamma(0)=\gamma(\ell)-\sum_{i=0}^{\ell-1} n(i) .
\end{aligned}
$$

Proof. Let $R_{i}$ be sum of exceptional curves that are contracted by $\mu_{i}$ for $0 \leq i<\ell$. From the pullback formula for the canonical class in $\S 2$ and from Lemma 1.a) it follows that $K_{i-1}=\mu_{i-1}^{*} K_{i}+R_{i-1}$ and $D_{i-1}=\mu_{i-1}^{*} D_{i}-K_{i-1}$. By abuse of notation we will denote $\mu_{i-1}^{*} D_{i}$ as $D_{i}$ and $\mu_{i-1}^{*} K_{i}$ as $K_{i}$.

It follows that $D_{\ell-1}=D_{\ell}-K_{\ell}-R_{\ell-1}$. In the next iteration we obtain $D_{\ell-2}=D_{\ell-1}-K_{\ell-1}-R_{\ell-2}=\left(D_{\ell}-K_{\ell}-R_{\ell-1}\right)-\left(K_{\ell}+R_{\ell-1}\right)-\left(R_{\ell-2}\right)=$ $D_{\ell}-2 K_{\ell}-2 R_{\ell-1}-R_{\ell-1}$. Repeating this we obtain

$$
D_{0}=D_{\ell}-\ell K_{\ell}-\sum_{i=0}^{\ell-1}(i+1) R_{i} \text {. }
$$

Similary we find

$$
K_{0}=K_{\ell}+\sum_{i=0}^{\ell-1} R_{i}
$$

From Lemma 2.c), it follows that $R_{i}^{2}=-n(i)$. From the projection formula in $\S 2$ it follows that $R_{i} \cdot R_{j}=D_{\ell} \cdot R_{i}=K_{\ell} \cdot R_{i}=0$ for $0 \leq i, j \leq \ell$.

The following algorithm outputs - for a given level, invariants of minimal ruled pair and the degree of the first ruled pair-invariants that follow the combinatorics of an adjoint chain. Moreover, the input level is as close as possible to the upper bound of Theorem 1 in terms of the invariants. The adjoint chain invariants in Example 1 were constructed with this algorithm and proof that the upper bounds of Theorem 1 are tight in a combinatorial sense. 


\section{Algorithm 1. (construct adjoint chain)}

input: Level $\ell, \alpha(\ell), \beta(\ell), \gamma(\ell)$ and $c \in \mathbb{Z}_{\geq 1}$.

output: The number of contracted curves $n(i)$ for $0 \leq i \leq \ell-1$ such that the difference between $\ell$ and the upper bound in Theorem 1 is minimal, under the condition that $\alpha(0)=c$. If the output is $\emptyset$, then no such valid adjoint chain exists for given input.

method: Below is the description of the algorithm in pseudo code using python syntax (\# is for commenting). The values $1, \mathrm{al}, \mathrm{bl}, \mathrm{gl}, \mathrm{c}$, None denote $\ell, \alpha(\ell), \beta(\ell), \gamma(\ell), c, \emptyset$ respectively. The function $\mathrm{a} 0(\mathrm{l}, \mathrm{al}, \mathrm{bl}, \mathrm{gl}, \mathrm{n})$ computes $\alpha(0)$ with the formula of Lemma 11.a). def construct_adjoint_chain( 1, al, bl, gl, c ):

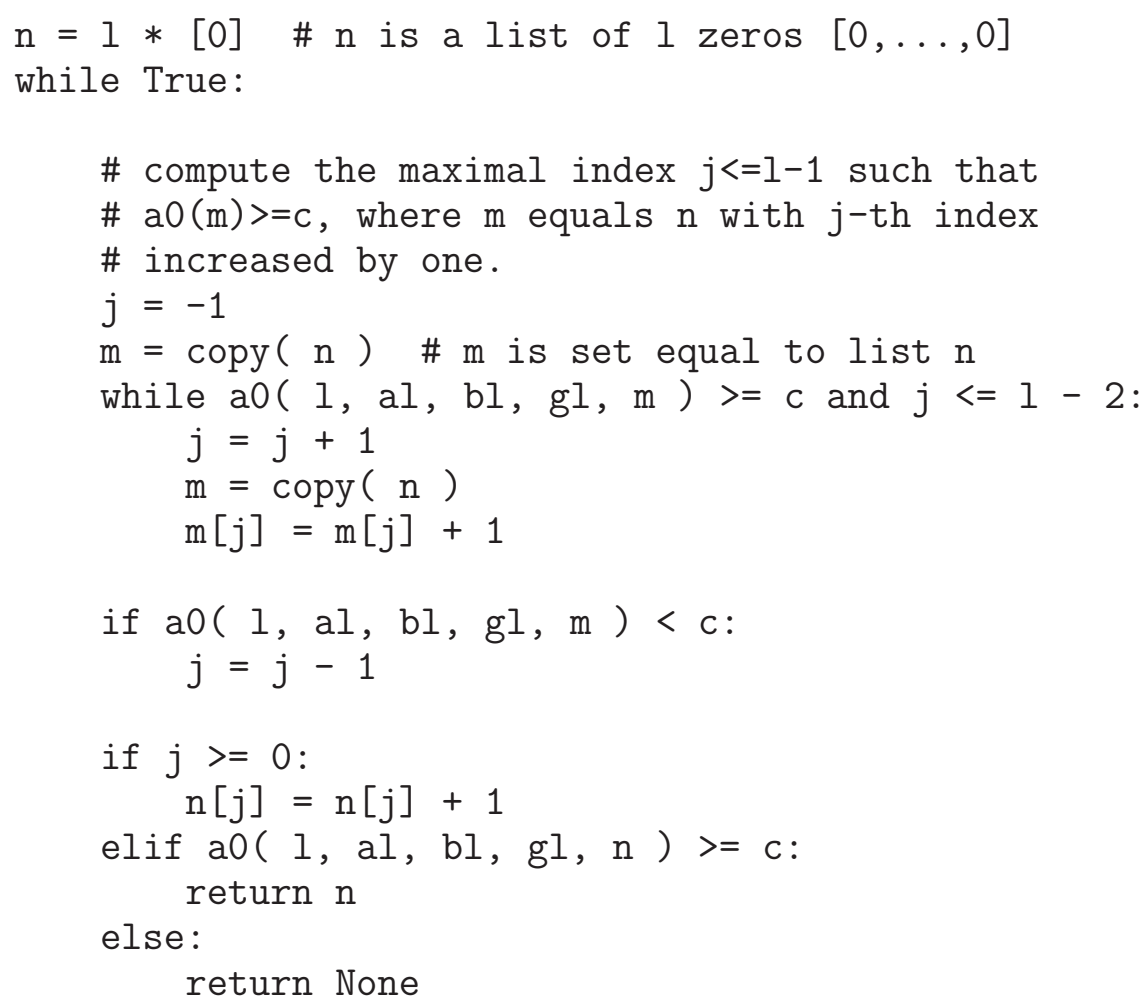




\section{Proposition 5. (algorithm)}

The output specification of Algorithm 1 is correct.

Proof. Note that the output and input of Algorithm 1 uniquely defines a sequence of invariants conform the rules of Lemma 2, Lemma 3 and Lemma 4:

$$
(n(i), \gamma(i), \beta(i), h(i), \alpha(i))_{0 \leq i \leq \ell-1} \text {. }
$$

In particular all the tables of Example 1 are constructed with Algorithm 1. We denote by $\tilde{\ell}$ the upper bound of Theorem 1 which depends on $\alpha(0), \beta(0)$ and $p$. From Proposition 2 we see that if $\gamma(\ell)>0$, then $p=0$ and otherwise $\gamma(\ell)=8(p+1)$.

From Lemma 11.a) it is immediate that the algorithm terminates. We prove that the algorithm outputs $n=(n(i))_{i}$, such that $\tilde{\ell}-\ell$ is minimal under the condition that $\alpha(0)=c$.

Claim 1: In order to minimize $\tilde{\ell}-\ell$ we need to minimize $\gamma(\ell)-\gamma(0)$ and maximize $\beta(0)$.

For the upper bound for $\ell$ as asserted in Lemma $6\left(S_{4}(0)\right)$ we assumed that $\gamma(0)=\ldots=\gamma(\ell)=1$ and $\beta(0)>0$. For the upper bound for $\ell$ as asserted in Lemma $7\left(S_{3}(0)\right)$ we assumed that $\gamma(0)=\ldots=\gamma(\ell-1)=0$ and $\beta(0)=\ldots=$ $\beta(\ell)=-1$. For the upper bound for $\ell$ as asserted in Lemma $8\left(S_{2}(0)\right.$ and $p \geq$ $-1)$ we assumed that $\gamma(0)=\ldots=\gamma(s-1)=-1, \gamma(s)=\ldots=\gamma(\ell-1)=0$ and $\beta(s)=\ldots=\beta(\ell)=-1$ such that $S_{2}(s-1)$ and $S_{3}(s)$. For the upper bound for $\ell$ as asserted in Lemma $9\left(S_{2}(0)\right.$ and $\left.p \leq-2\right)$ we assumed that $\gamma(0)=\ldots=\gamma(\ell)$. It follows - under the constraints of Lemma 3 - that in order to minimize $\tilde{\ell}-\ell$ we want to minimize $\gamma(\ell)-\gamma(0)$ and maximize $\beta(0)$. This completes the proof of claim 1.

From Lemma 11.c) we find that we minimize $\gamma(\ell)-\gamma(0)$ if we minimize:

$$
\Gamma:=\sum_{i=0}^{\ell-1} n(i) .
$$


From Lemma 11.b) we find that we maximize $\beta(0)$ if we maximize:

$$
\Theta:=\sum_{i=0}^{\ell-1}(i+1) n(i) .
$$

From Lemma 11.a) we find that $\alpha(0)=C-\Lambda$ where

$$
\Lambda:=\sum_{i=0}^{\ell-1}(i+1)^{2} n(i),
$$

and $C$ is a constant which depends on the input. If $C<c$, then the algorithm returns $\emptyset$. Otherwise, we ensure that $\alpha(0)=c$ with the $n(0)$ term in $\Lambda$.

At each step of the while-loop the algorithm increases $n(i)$ with one, for as large possible $i$, under the constraint that $\alpha(0) \geq c$. This way $\Theta$ is maximized since the coefficient of $n(i)$ is $i+1$. The term $\Lambda$ is maximized even more since the coefficient of $n(i)$ equals $(i+1)^{2}$. Therefore the condition $\alpha(0)=c$ is met in a minimal number of steps. Thus $\Gamma$ is minimized under the constraint that $\alpha(0) \geq c$. Now it follows from claim 1 that the output specification of Algorithm 1 is correct.

\subsection{Geometric meaning of the constant c}

Suppose that the adjoint chain of $\left(X_{0}, D_{0}\right)$ has invariants conform input and output of Algorithm 1. From Proposition 2 it follows that the arithmetic genus of $X_{0}$ equals $p=\min \left(0,\left\lceil\frac{1}{8} \gamma(\ell)-1\right\rceil\right)$.

The input constant $c$ equals the degree of the polarized model of $\left(X_{0}, D_{0}\right)$.

We will now argue that the constant $c$ also measures the embedding dimension of the polarized model of $\left(X_{0}, D_{0}\right)$. Recall that the embedding dimension of the polarized model of $\left(X_{0}, D_{0}\right)$ equals $h^{0}\left(D_{0}\right)-1$. If $D_{0}$ is ample, then it follows from Kodaira vanishing theorem and Riemann Roch theorem that

$$
h^{0}\left(D_{0}\right)=\frac{h(0)}{2}+p+1
$$


By increasing the input constant $c$ we increase $h(0)$ and consequently $h^{0}\left(D_{0}\right)$. If $D_{0}-K_{0}$ is only nef and big, then alternatively we can use the KawamataViehweg vanishing theorem.

Recall that by definition of nef and big only a high enough multiple of $D_{1}$ defines a birational morphism. Reider's theorem [1, Theorem 11.4] says that if $c=D_{0}^{2} \geq 10$ and there exists no curve $C$ such that $\left(D_{0} \cdot C=0\right.$ and $\left.C^{2}=-1\right)$ or $\left(D_{0} \cdot C=1\right.$ and $\left.C^{2}=0\right)$ or $\left(D_{0} \cdot C=2\right.$ and $\left.C^{2}=0\right)$, then $D_{0}+K_{0}$ defines a birational morphism. Notice that $D_{1}$ is the pushforward of $D_{0}+K_{0}$ by definition and thus if $D_{0}+K_{0}$ defines a birational morphism, then the polarized model of $\left(X_{1}, D_{1}\right)$ is a surface.

\subsection{Computing examples from output of algorithm}

Let input $\ell, \alpha(\ell), \beta(\ell), \gamma(\ell), c$ and output $(n(i))_{0 \leq i \leq \ell-1}$ of Algorithm 1 be given. The output of Algorithm 1 is not necessarily geometric in the sense that $\left(X_{0}, D_{0}\right)$ exists such that the polarized model of $\left(X_{0}, D_{0}\right)$ is a surface. In particular, $\alpha(\ell), \beta(\ell), \gamma(\ell)$ has to be conform Proposition 2. However, if the output is geometric, then we can compute - at least in theory-equations for a polarized model of $\left(X_{0}, D_{0}\right)$ such that its adjoint chain has the corresponding invariants.

For the sake of simplicity we assume that $X_{\ell}$ is the blowup of the projective plane with $D_{\ell}=-K_{\ell}$ as in Proposition 2. We blow up $X_{\ell}$ in $n(\ell-1)$ generic points. It follows from Lemma 1.a) and the pullback formula for the canonical class in $\S 2$ that

$$
D_{\ell-1}=\mu^{*} D_{\ell}-\mu^{*} K_{\ell}+\sum_{i=1}^{n(\ell-1)} E_{i}^{\prime},
$$

where $E_{i}^{\prime}$ are disjoint exceptional curves. Similar as in the proof of Lemma 11, we find after sequentially taking the pullback as above that

$$
D_{0}=d H-\sum_{j} m_{j} E_{j}
$$


where $H$ is the pullback of lines in the projective plane, $m_{j}>0$ and the $E_{j}$ are the pullback of exceptional curves. Note that $D_{0}^{2}=c$ by assumption.

We construct a linear series $\left|D_{0}\right|$ in the plane with polynomials of degree $d$ and generic base points with multiplicities $\left(m_{i}\right)_{i}$. We check whether the map associated to the linear series parametrizes a surface, otherwise we have to consider a multiple of $D_{0}$ (see $\left.\S 6.2\right)$. After a generic projection we may assume that we have a parametrization of a hypersurface in 3-space. We consider an implicit equation of degree $\alpha(0)$ with undetermined coefficients and substitute the parametrization. We obtain an implicit equation by solving the linear system of equations in the undetermined coefficients.

See [8, Example 52] for worked out equations for a surface of degree 8 with a minimal family of degree 8. As illustrated in Table 1 of Example 1, a surface of degree 8 has minimal family degree of at most 18 .

\section{$7 \quad$ Inequality for lattice polygons}

Let $\left(X_{0}, D_{0}\right)$ be a toric surface with polarized model $Y_{0} \subset \mathbb{P}^{n}$. We define the lattice polygon $P_{0}$ by taking the convex hull of the lattice points in the lattice $\mathbb{Z}^{2} \subset \mathbb{R}^{2}$ with coordinates defined by the exponents of a monomial parametrization $\left(\mathbb{C}^{*}\right)^{2} \longrightarrow Y_{0}$.

We denote $\rho(0)$ for the Picard number of $X_{0}$. We define $S(0)$ to be the number of exceptional divisors in the minimal resolution of the isolated singularities of $Y_{0}$. We introduce the following notation:

$$
v(0):=\rho(0)+2-S(0) .
$$

The adjoint of a lattice polygon is defined as the convex hull of its interior lattice points. We call a lattice polygon minimal if its adjoint is either the empty set, a point or a line segment. The level $\ell\left(P_{0}\right)$ of a lattice polygon is defined as the number of subsequent adjoint lattice polygons $P_{0} \longrightarrow \ldots \longrightarrow P_{\ell\left(P_{0}\right)}$ until 
a minimal lattice polygon $P_{\ell\left(P_{0}\right)}$ is obtained. See Remark 1 concerning the alternative definition for level as in [11, Section 2.3] and [5, Section 3].

We recall part of the dictionary in [6, Section 1] using the notation at the beginning of $\S 5.1$ :

- $\frac{\alpha(0)}{2}=a\left(P_{0}\right)$ where $a\left(P_{0}\right)$ is the area of $P_{0}$,

- $-\beta(0)=b\left(P_{0}\right)$ where $b\left(P_{0}\right)$ is the number of boundary lattice points,

- $v(0)=v\left(P_{0}\right)$ where $v\left(P_{0}\right)$ is the number of vertices of $P_{0}$, and

- $\ell=\ell\left(P_{0}\right)$ where $\ell\left(P_{0}\right)$ is level of $P_{0}$.

From Lemma 11 it follows that

$$
\alpha(0)+2 \ell \beta(0)=-\gamma(\ell) \ell^{2}+\alpha(\ell)+\Phi
$$

where

$$
\Phi:=\sum_{i=0}^{\ell-1}(2 \ell-i-1)(i+1) n(i) .
$$

As an immediate consequence we obtain the following inequality

$$
\alpha(0)+2 \ell \beta(0)+\gamma(\ell) \ell^{2} \geq 0
$$

From Proposition 2 it follows that $\gamma(\ell) \leq 9$ and by substituting 9 for $\gamma(\ell)$ in (2) we recover the inequality of [6, Theorem 5]. Moreover, we see that the inequality holds more generally for birationally ruled surfaces. Note that for irrational birationally ruled surfaces we have that $\gamma(\ell) \leq 0$.

We want improve (2) by bounding $\Phi$ in terms of $v(0)$. From Proposition 2 it follows that $\rho(\ell) \leq 9$. We recall from $\S 2$ that the Picard number decreases by 1 for each contracted exceptional curve, and thus

$$
\sum_{i=0}^{\ell-1} n(i) \geq \rho(0)-9 \geq v(0)-11 .
$$


From $(2 \ell-i-1)(i+1) \geq 2 \ell-1$ for all $0 \leq i \leq \ell-1$ it follows that

$$
\Phi \geq(2 \ell-1) \sum_{i=0}^{\ell-1} n(i) \geq(2 \ell-1)(v(0)-11) .
$$

Now from (1), (3) and $\alpha(\ell) \geq 0$ we obtain the following inequality on invariants of birationally ruled surfaces:

\section{Theorem 2.}

$$
\alpha(0)+2 \ell \beta(0)+9 \ell^{2} \geq(2 \ell-1)(v(0)-11) .
$$

Restricting to toric surfaces and applying the dictionary we obtain an improved inequality for lattice polygons, as was conjectured in [6, Section 4], [3, Section 2.7]:

\section{Corollary 2.}

$$
2 a\left(P_{0}\right)-2 \ell\left(P_{0}\right) b\left(P_{0}\right)+9 \ell\left(P_{0}\right)^{2} \geq\left(2 \ell\left(P_{0}\right)-1\right)\left(v\left(P_{0}\right)-11\right) .
$$

\section{Acknowledgements}

I would like to thank Josef Schicho for useful discussions. This work was

supported by base funding of the King Abdullah University of Science and Technology.

\section{Bibliography}

[1] W.P. Barth, K. Hulek, C. A. M. Peters, and A. Van de Ven. Compact complex surfaces. Springer-Verlag, Berlin, second edition, 2004.

[2] A. Beauville. Complex algebraic surfaces. Cambridge University Press, 1983. 
[3] W. Castryck. Moving out the edges of a lattice polygon. Discrete Comput. Geom., 47(3):496-518, 2012.

[4] I. V. Dolgachev. Classical algebraic geometry: A modern view. Cambridge University Press, Cambridge, 2012.

[5] C. Haase and J. Schicho. Lattice polygons and the number $2 i+7$. Amer. Math. Monthly, 116(2):151-165, 2009.

[6] C. Haase and J. Schicho. An inequality for adjoint rational surfaces. arXiv:1306.3389, 2013.

[7] N. Lubbes. Minimal families of curves on surfaces. Journal of Symbolic Computation, 2013.

[8] N. Lubbes and J. Schicho. Lattice polygons and families of curves on rational surfaces. Journal of Algebraic Combinatorics, pages 1-24, 2010.

[9] K. Matsuki. Introduction to the Mori program. Universitext. SpringerVerlag, New York, 2002.

[10] J. Schicho. A degree bound for the parameterization of a rational surface. J. Pure Appl. Alg., 145:91-105, 2000.

[11] J. Schicho. The parametric degree of a rational surface. Math. Z., 254: 185-198, 2006.

address of author: Johann Radon Institute for Computational and Applied Mathematics (RICAM), Austrian Academy of Sciences

email: niels.lubbes@gmail.com 